\title{
An unusual case of malrotation in a baby conceived by in vitro fertilization
}

\begin{abstract}
Introduction: Interruption of intestinal rotation and fixation during any point in the development of the midgut results in an anatomic spectrum encompassed by the term 'malrotation'.

Case presentation: We present an unusual variant of malrotation in a neonate conceived by In vitro fertilization.

Discussion: Assisted reproductive techniques have been recognized to be associated with a higher risk of congenital anomalies. However, there have been inconsistent reports regarding an increased risk of gastrointestinal anomalies. Recent reports have also linked assisted reproductive techniques to an increased risk of genetic imprinting disorders. There is evidence to suggest that some cases of malrotation may have a genetic aetiology. The fact that the case presented was conceived by In vitro fertilization may be entirely incidental, however the unusual bowel orientation has led us to speculate whether assisted reproductive techniques might be associated with rarer forms of malrotation.
\end{abstract}

Keywords: malrotation, assisted reproductive techniques, in vitro fertilization
Volume I Issue 5 - 2014

\section{Caroline Pardy}

The Royal Alexandra Children's Hospital, UK

\author{
Correspondence: Caroline Pardy, Royal Alexandra \\ Children $\square$ s Hospital, Brighton, UK,
}

Email carolinepardy@gmail.com

Received: July 15, 2014 | Published: October 06, 2014
Abbreviations: SMA, superior mesenteric artery; IVF, in vitro fertilization; ISCI, intracytoplasmic sperm injection

\section{Introduction}

Rotation of the midgut occurs between 4-12 weeks of embryological life. Between 4-10 weeks, the midgut herniates through the umbilicus. The duodenojejunal loop initially lies above the superior mesenteric artery (SMA), and undergoes a 270 degree counter-clockwise rotation, coming to lie below, initially to the right and finally to the left of the SMA. Between 10-11 weeks, the midgut returns to the abdomen, and the caecocolic loop undergoes a 270 degree counterclockwise rotation. The caecocolic loop initially lies beneath the SMA, but rotates to lie to the left, then above and finally to the right of the SMA. Retroperitoneal fixation of the duodenojejunal flexure and the caecum occurs after 11 weeks. This results in a broad-based mesentery extending from the ligament of Treitz to the ileocaecal junction, preventing midgut volvulus.

'Malrotation' is a term that has been applied to a spectrum of abnormal intestinal position and fixation. As a result, the mesentery has a narrowed pedicle, predisposing to midgut volvulus. The incidence of intestinal malrotation is said to be $0.2 \%$ ( 1 in 500). ${ }^{1}$ In a 25 -year review of malrotation at a single institution, $31 \%$ presented within the first week of life, and $53 \%$ within the neonatal period. ${ }^{2}$ Classic neonatal presentation is with bilious vomiting and signs of high intestinal obstruction. Patients presenting in the first few days of life were noted to be at greatest risk for associated volvulus and resulting necrotic bowel.

Diagnosis is most commonly made using an upper gastrointestinal contrast study. Malrotation is suggested if the duodenojejunal junction is seen to be to the right of the spine, or in the midline. Ultrasound can also be used to support a diagnosis of malrotation by demonstrating inversion of the normal position of the superior mesenteric artery and vein.
Ladd $^{3}$ described the definitive treatment of malrotation still practised today. If midgut volvulus is encountered, counter-clockwise detorsion is undertaken. Viability of the bowel is assessed, and the bowel is examined to exclude an associated duodenal atresia or web. Mesenteric bands (Ladd's bands), tethering the caecum across the duodenum, are divided, and the mesentery is broadened. The bowel is placed with small bowel on the right, and large bowel on the left. An incidental appendicectomy is usually performed, to avoid future confusion due to its altered position.

\section{Case presentation}

A term male infant conceived via In vitro fertilisation, presented with bile-stained vomiting 2 days after discharge from the maternity unit. Observations on admission were within normal parameters, and the abdomen was non-distended. An abdominal film revealed duodenal and proximal jejunal distension, with paucity of small bowel gas distally (Figure 1). The patient was taken to theatre with a tentative diagnosis of jejunal stenosis.

Laparotomy revealed a complex pattern of malrotation, with proximal jejunum obstructed at the point of passage through the mesentery of the distal ileum (Figure 2). De-rotation was not possible without the division of the principle vascular arcade supplying the small bowel. As this would have significantly compromised the bowel, the defect in the small bowel mesentery at the point of the stenosis was widened, and the bowel left in its abnormal orientation (Figure 3). The baby had an uncomplicated post-operative recovery and was discharged within a week on full feeds. He has remained well, and is thriving 2 years following his surgery.

\section{Discussion}

Internal hernias are recognized as a rare feature of some forms of malrotation. Left and right paraduodenal hernias are the most common recognized internal hernias, with left being more common than right. ${ }^{4}$ A right paraduodenal hernia results from incomplete rotation 
resulting in the small bowel becoming caught in the mesentery of the ascending colon. When the ascending colon becomes fixed to the retroperitoneum, the small bowel loops become trapped behind the mesentery. A left paraduodenal hernia is thought to result from reversed rotation, resulting in the small bowel becoming trapped by the mesentery of the descending colon upon its retroperitoneal fixation. ${ }^{5}$ The findings at laparotomy in this case are clearly different to the paraduodenal hernias described in the literature. As demonstrated in Figure 2, rather than large bowel mesentery, it is at the level of the small bowel mesentery where jejunal obstruction occurs.

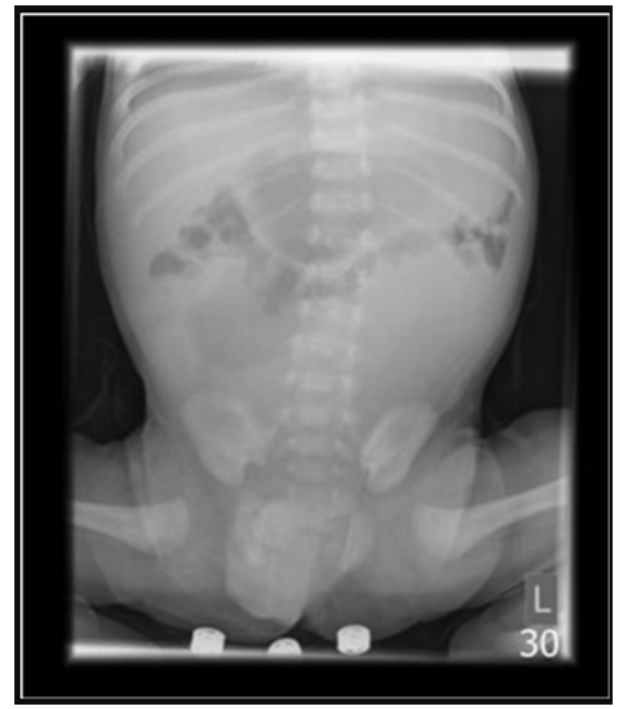

Figure I Abdominal radiograph demonstrating distended stomach and proximal small bowel with paucity of distal gas, suggestive of jejunal stenosis.

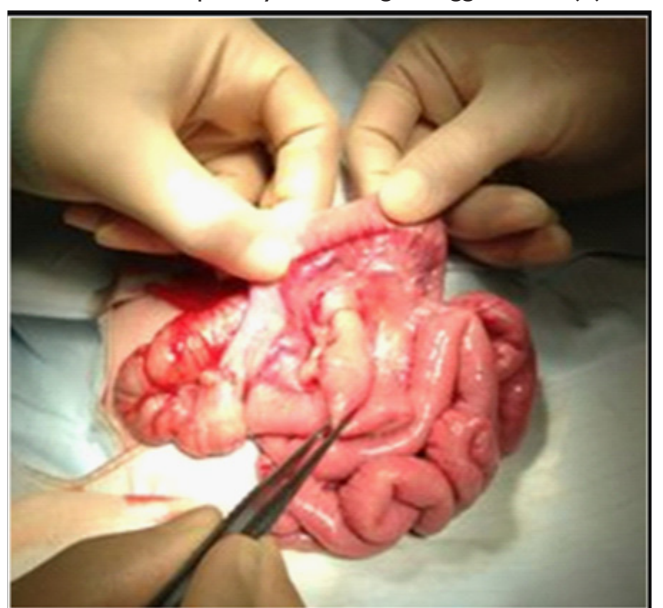

Figure 2 Findings at laparotomy; obstruction of proximal jejunum at point of passage through defect in small bowel mesentery.

Assisted reproductive techniques have been acknowledged to be associated with a higher risk of congenital anomalies. A metaanalysis of 46 studies incorporating 124468 babies conceived using In vitro fertilization (IVF) and intracytoplasmic sperm injection (ICSI), identified a relative risk of an associated congenital anomaly of 1.37 compared to spontaneously conceived babies. ${ }^{6}$ A higher, but not statistically significant risk of congenital anomalies was found for babies conceived using ICSI compared to IVF. The malformations considered to be more prevalent with assisted reproductive techniques include central nervous system anomalies, cardiovascular defects, kidney agenesis or dysgenesis, limb reduction defects and congenital malformation syndromes. Debate appears to exist regarding a possible increased risk of gastrointestinal anomalies. A Swedish study of congenital malformations associated with IVF observed an increased risk of oesophageal atresia, small bowel and anal atresia, in their data analyzed from 1982-2001. ${ }^{7}$ However, analysis of data from 2001-2007 by the same group found only oesophageal atresia to be significantly increased. A German study however, noted an increased risk of anorectal malformation with assisted reproduction. ${ }^{8}$ A review of the literature does not suggest that malrotation has been identified as being more prevalent in babies conceived via assisted reproductive techniques.

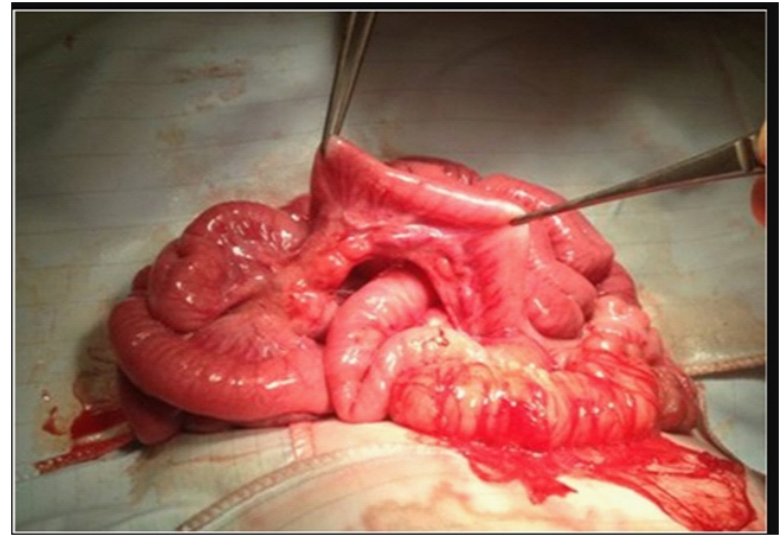

Figure 3 Relief of obstruction by widening the defect the small bowel mesentery.

Concerns have also been raised regarding a possible increased risk of imprinting disorders including Beckwith Wiedemann syndrome, Angelman syndrome and retinoblastoma. ${ }^{9}$ Genetics are thought to play an important role in malrotation, ${ }^{10}$ with individual gene mutations such as the forkhead box transcription factor $(F o x f 1)^{11}$ being implicated. Furthermore, evidence for autosomal dominant inheritance in familial non-syndromic intestinal malrotation ${ }^{12,13}$ and syndromic intestinal malrotation, have been described. With the suggestion that assisted reproductive techniques could be associated with an increased risk of genetic disruption, it is not inconceivable that an increased risk of malrotation may be observed.

We have described an unusual case of malrotation, whose form does not appear to have been described previously in the literature. This case emphasizes the broad spectrum of abnormal bowel rotation encompassed by the term 'malrotation', and the need for the surgeon to adapt their approach to definitive management when faced with such challenging cases. The fact that this baby was conceived by assisted reproduction may be coincidental, however it should raise awareness that unusual forms of malrotation may be observed with the increased use of assisted reproductive techniques.

\section{Acknowledgments}

None.

\section{Conflicts of Interest}

There is no conflict of interest.

\section{Funding}

None.

\section{References}

1. Kantor JL. Anomalies of the colon: their Roentgen diagnosis and clinical significance. Radiology. 1934;23(6):651-662. 
2. Andrassy RJ, Mahour GH. Malrotation of the midgut in infants and children: a 25-year review. Arch Surg. 1981;116(2):158-160.

3. Ladd WE. Surgical diseases of the alimentary tract in infants. $N$ Engl $J$ Med. 1936;215(16):705-708.

4. Strouse PJ. Disorders of intestinal rotation and fixation ('malrotation') Pediatr Radiol. 2004;34(11):837-851.

5. Zimmerman LM, Laufman H. Intra-abdominal hernias due to developmental and rotational anomalies. Ann Surg. 1953;138(1): 82-91.

6. Wen J, Jiang J, Ding C, et al. Birth defects in children conceived by in vitro fertilization and intracytoplasmic sperm injection: a meta-analysis. Fertil Steril. 2012;97(6):1331-1337.

7. Kallen B, Finnstrom O, Lindam A, et al. Congenital malformations in infants born after in vitro fertilization in Sweden. Birth Defects Res A CLin Mol Teratol. 2010;88(3):137-143.
8. Zwink K, Jenetzky E, Schmiedeke E, et al. Assisted reproductive techniques and the risk of anorectal malformations: a German casecontrol study. Orphanet J Rare Dis. 2012;15(7):65.

9. Manipalviratn S, DeCherney A, Segars J. Imprinting disorders and assisted reproductive technology. Fertil Steril. 2009;91(2):305-315.

10. Martin V, Shaw-Smith C. Review of genetic factors in intestinal malrotation. Pediatr Surg Int. 2010;26(8):769-781.

11. Stankiewicz P, Sen P, Bhatt SS, et al. Genomic and genic deletions of the FOX gene cluster on 16q24.1 and inactivating mutations of FOXF1 cause alveolar capillary dysplasia and other malformations. Am J Hum Genet. 2009;84(6):780-791.

12. Smith SL. Familial midgut volvulus. Surgery. 1972;72(3):420-426.

13. Beaudoin S, Mathiot-Gavarin A, Gouizi G, et al. Familial malrotation: report of three affected siblings. Pediatr Surg Int. 2005;21(10):856-857. 